\title{
A!
}

This is an electronic reprint of the original article.

This reprint may differ from the original in pagination and typographic detail.

Räsänen, E.; Könemann, J.; Haug, R.J.; Puska, M.J.; Nieminen, R.M.

\section{Impurity effects in quantum dots: Toward quantitative modeling}

Published in:

Physical Review B

DOI:

10.1103/PhysRevB.70.115308

Published: 14/09/2004

Document Version

Publisher's PDF, also known as Version of record

Please cite the original version:

Räsänen, E., Könemann, J., Haug, R. J., Puska, M. J., \& Nieminen, R. M. (2004). Impurity effects in quantum dots: Toward quantitative modeling. Physical Review B, 70(11), 1-6. [115308].

https://doi.org/10.1103/PhysRevB.70.115308

This material is protected by copyright and other intellectual property rights, and duplication or sale of all or part of any of the repository collections is not permitted, except that material may be duplicated by you for your research use or educational purposes in electronic or print form. You must obtain permission for any other use. Electronic or print copies may not be offered, whether for sale or otherwise to anyone who is not an authorised user. 


\title{
Impurity effects in quantum dots: Toward quantitative modeling
}

\author{
E. Räsänen, ${ }^{1, *}$ J. Könemann, ${ }^{2}$ R. J. Haug, ${ }^{2}$ M. J. Puska, ${ }^{1}$ and R. M. Nieminen ${ }^{1}$ \\ ${ }^{1}$ Laboratory of Physics, Helsinki University of Technology, P.O. Box 1100, FIN-02015 HUT, Finland \\ ${ }^{2}$ Institut für Festkörperphysik, Universität Hannover, Appelstrasse 2, D-30167 Hannover, Germany
}

(Received 23 April 2004; published 14 September 2004)

\begin{abstract}
We have studied the single-electron transport spectrum of a quantum dot in GaAs/AlGaAs resonant tunneling device. The measured spectrum has irregularities indicating a broken circular symmetry. We model the system with an external potential consisting of a parabolic confinement and a negatively charged Coulombic impurity placed in the vicinity of the quantum dot. The model leads to good agreement between the calculated single-electron eigenenergies and the experimental spectrum. Furthermore, we use the spin-density-functional theory to study the energies and angular momenta when the system contains many interacting electrons. In the high magnetic field regime the increasing electron number is shown to reduce the distortion induced by the impurity.
\end{abstract}

DOI: 10.1103/PhysRevB.70.115308

PACS number(s): 73.63.Kv, 73.21.La, 71.55.Eq, 75.75.+a

\section{INTRODUCTION}

The tunability in size, shape, and electron number of semiconductor quantum dots (QD) provides numerous technological applications as well as interesting many-electron physics. ${ }^{1}$ In actual QD devices, the effects induced by impurities or donor scattering centers may be remarkable. In most cases, irregularities in samples have only an indirect influence on the many-body structure, complicating the identification of the origin behind the peculiar behavior in the measured characteristics of QD's.

A clean quantum dot typically shows single-electron energy levels reminiscent of the well-known Fock-Darwin energy spectrum corresponding to a parabolic confining potential. ${ }^{2}$ Adding external impurities into the QD breaks the circular symmetry of the system, leading to avoided crossings and liftings of the degeneracies in the single-electron energy spectrum. This was demonstrated by Halonen et al., ${ }^{3}$ who studied theoretically QD's distorted by repulsive Gaussian scattering centers. However, even if clear traces of the Fock-Darwin spectrum have been obtained experimentally in both lateral ${ }^{4}$ and vertical ${ }^{5-7}$ quantum dots, there is, to the best of our knowledge, no direct experimental evidence of repulsive impurities present in QD structures. Instead, states bound to hydrogenic impurities, probably arising from $\mathrm{Si}$ dopant atoms in the GaAs quantum well, were found already by Ashoori and co-workers ${ }^{8}$ in their pioneering singleelectron tunneling experiment. These impurities have been suggested to be sources of pair-tunneling states, theoretically analyzed with a superimposed attractive $1 / r$-type potential.., 10

Theoretically, the distortion of the circular symmetry makes the many-electron problem particularly complex to solve, especially in the presence of an external magnetic field. In the above-mentioned study, Halonen et al. ${ }^{3}$ applied exact diagonalization up to three electrons and focused on the effects of impurities on the energy levels and optical absorption spectra. Recently, Güçlü and co-workers ${ }^{11}$ performed diffusion quantum Monte Carlo calculations on QD's distorted by randomly distributed Gaussian scatterers and studied the energetics up to ten electrons. They found that in these systems the transitions between the many-body states are considerably less pronounced than in clean dots. Hirose and Wingreen ${ }^{12}$ have used the spin-density-functional theory (SDFT) to examine the energies and spin states in disordered QD's as a function of the interaction strength in zero magnetic field. Besides additional scatterers, noncircular QD's have attracted general interest in connection with the chaotic propertie ${ }^{13}$ or the behavior in the high magnetic field limit. ${ }^{14}$

In this paper we present a measured single-electron transport spectrum where avoided crossings and lifted degeneracies are clearly observable. We reproduce the spectrum with an appropriate model potential, showing that the unexpected effects in the spectrum result from a negatively charged Coulombic impurity located near the QD. The many-electron properties studied by the SDFT reflect the strongly distorted single-electron spectrum. The variation of the impurity location shows the stability of the maximum-density droplet (MDD) and the screening of the impurity by electrons.

The outline of this paper is as follows. In Sec. II we briefly describe the fabrication of the sample and report the transport measurement. In Sec. III the theoretical model describing the physical system is given and the single-electron calculations are compared to the experiment. In Sec. V the many-electron properties, i.e., chemical potentials, MDD stability, and total magnetization are studied with the SDFT. The paper is summarized in Sec. VI.

\section{EXPERIMENT}

The heterostructure consists of a $10 \mathrm{~nm}$ wide GaAs quantum well sandwiched between two $\mathrm{Al}_{0.3} \mathrm{Ga}_{0.7}$ As-tunneling barriers of 5 and $8 \mathrm{~nm}$, see Fig. 1. The contacts are formed by $0.5 \mu \mathrm{m}$ thick GaAs layers highly doped with $\mathrm{Si}$ up to 4 $\times 10^{17} \mathrm{~cm}^{-3}$ and separated from the active region by $7 \mathrm{~nm}$ thin spacer layers of undoped GaAs. Our sample was defined as a mesa of $40 \mu \mathrm{m}$ size. We carried out direct-current measurements of the current-voltage $(I-V)$ characteristics in a $\mathrm{He}^{3}$-refrigerator at $350 \mathrm{mK}$ base temperature in magnetic fields up to $14 \mathrm{~T}$. 


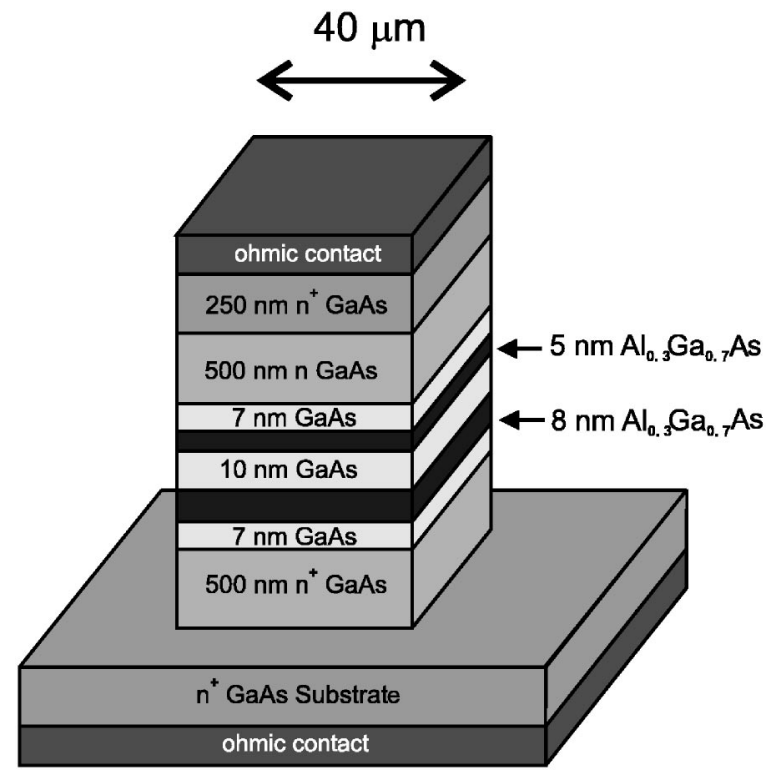

FIG. 1. Sketch of the heterostructure of our sample.

Figure 2 shows the resulting transport spectrum of a quantum dot formed in a local potential minimum. The black lines $V_{n, l}$ correspond to high differential conductance $G$ $=\mathrm{d} I / \mathrm{d} V$. They trace the position of the single-electron energy states $E_{n, l}$ of the spectrum according to a relation

$$
V_{n, l}=V_{0}+1 /(e \alpha) E_{n, l}
$$

where the energy-voltage conversion factor $\alpha$ equals 0.4 , determined from measurements of the broadening of the step edge with temperature, and the onset voltage is fitted to be $V_{0}=172 \mathrm{mV}$. The energy levels $V_{n, l}$ in the transport spectrum can be interpreted as single-electron energies of a local, presumably a growth-induced potential minimum in the GaAs quantum well of our device. Several energy levels are cleary visible in Fig. 2. In contrast to ordinary Fock-Darwin energy levels, we are able to observe broken energy degeneracies at $B=0 \mathrm{~T}$ and strong anticrossing effects in the spectrum.

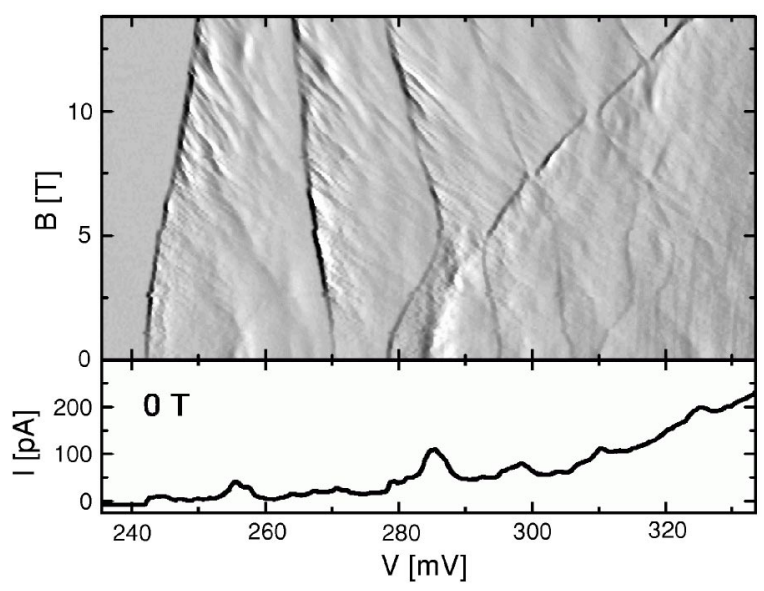

FIG. 2. Top: $G(V, B)$ plot of the transport spectrum of our sample. Bottom: $I-V$ characteristics for $B=0 \mathrm{~T}$.

\section{MODELING THE QUANTUM DOT}

We expect the quantum well confined in the GaAs layer to have a negligible degree of freedom for electrons in the vertical direction. Our model system is thus strictly twodimensional (2D) and defined to be located on the $x y$ plane. The single-electron Hamiltonian is written as

$$
h(\mathbf{r})=\frac{1}{2 m^{*}}[\mathbf{p}+e \mathbf{A}(\mathbf{r})]^{2}+V_{\text {conf }}(\mathbf{r})+V_{\text {imp }}(\mathbf{r}),
$$

where we use the effective-mass approximation (EMA) with $m^{*}=0.067 m_{e}$, which is the typical value for electrons moving in GaAs. In a symmetric gauge the vector potential reads as $\mathbf{A}=B / 2(-y, x, 0)$, giving the external magnetic field $\mathbf{B}$ $=B \hat{z}$ perpendicular to the QD plane. The Zeeman energy is omitted in Eq. (2) since the spin splitting ${ }^{15}$ is not visible in the energy levels shown in Fig. 2 for the magnetic fields applied.

The confining potential $V_{\text {conf }}(\mathbf{r})$ is expected to be parabolic near the center of the dot. However, we soften the edges of the dot by changing the sign of the paraboloid at a certain cusp radius $r_{c}$, giving

$$
V_{\text {conf }}(\mathbf{r})=\left\{\begin{array}{l}
\frac{1}{2} m^{*} \omega_{0}^{2} r^{2}, \quad r \leqslant r_{c} \\
m^{*} \omega_{0}^{2}\left[s\left(r-r_{c}\right)^{2}-r_{c}\left(\frac{r_{c}}{2}-r\right)\right], \quad r>r_{c},
\end{array}\right.
$$

where the parameter $s$ defines the strength of the rounding term. As shown below, the softening of the confinement is crucial in obtaining a good agreement with the experimental energy spectrum.

We expect the impurity to be described by a negatively charged particle located in the vicinity of the quantum well. The impurity potential can thus be written in a Coulombic form as

$$
V_{\mathrm{imp}}(\mathbf{r})=\frac{e|q|}{4 \pi \epsilon_{0} \epsilon \sqrt{(\mathbf{r}-\mathbf{R})^{2}+d^{2}}},
$$

where $q$ is the (negative) charge of the impurity particle, $\epsilon$ $=12.7$ is the dielectric constant for GaAs, and $R$ and $d$ are the lateral and vertical distances of the impurity from the QD center, respectively. Figure 3 shows the total external confinement of the model system, $V_{\text {ext }}=V_{\text {conf }}+V_{\text {imp }}$, and a sketch of the expected configuration.

To calculate the single-electron spectrum, we solve the discretized eigenvalue problem $h \psi_{i}=\epsilon_{i} \psi_{i}$ numerically on a two-dimensional (2D) point grid using a Rayleigh quotient multigrid method. ${ }^{16}$ Figure 4 shows the resulting spectrum (dashed lines) compared to the experimental data (repeated from Fig. 2). The energies are converted to voltages according to Eq. (1), and the model parameters are adjusted (see the discussion below) until the agreement between the experiment and the model is as good as possible. The simulation places the avoided crossings between the energy levels very close to the correct positions. There are still considerable deviations in the 5th and 6th levels but, for example, the 7th level agrees almost perfectly through the magnetic-field regime presented. The differences at high fields between the 


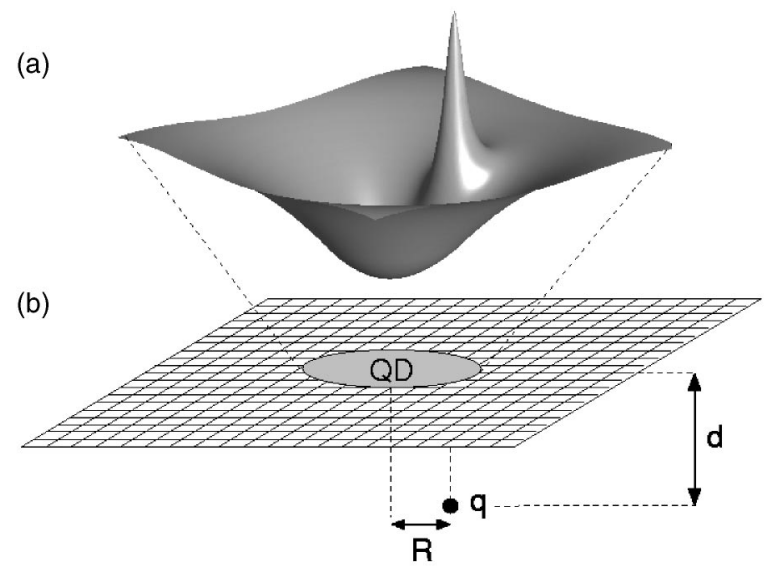

FIG. 3. (a) Profile of the external potential used in the simulation. (b) Sketch of the expected configuration of the QD-impurity system.

experimental data and the simulation result from the shift of the chemical potential of the emitter to higher energies with increasing magnetic field.

In calculating the energy spectrum shown in Fig. 4, we optimize the potential parameters corresponding to the best possible fit to the experimental data. The confinement is then defined by $\hbar \omega_{0}=13.8 \mathrm{meV}, r_{c}=15.5 \mathrm{~nm}$, and $s=-0.2$, and the impurity parameters are given by $q=-2 e, R=14.5 \mathrm{~nm}$, and $d=2 \mathrm{~nm}$. There is naturally some uncertainty in the parameters due to the rather similar scaling of $V_{\text {ext }}$ with respect to $|q|$ and $d$. In addition, the exact value for $\epsilon$ describing the screening of the impurity is not known. We used the simplest assumption, i.e., the same value for $\epsilon$ that is applied with the EMA to screen the electron-electron interactions for the 2D electron gas in GaAs. This fixation yields $q=-2 e$ for the best fit. In reality, however, the screening may be reduced so that a single impurity charge is also possible.

Thus, for this particular sample we determine the following characteristic features of the system from the model potential $V_{\text {ext }}$ : (i) The impurity is presumably an ionized single

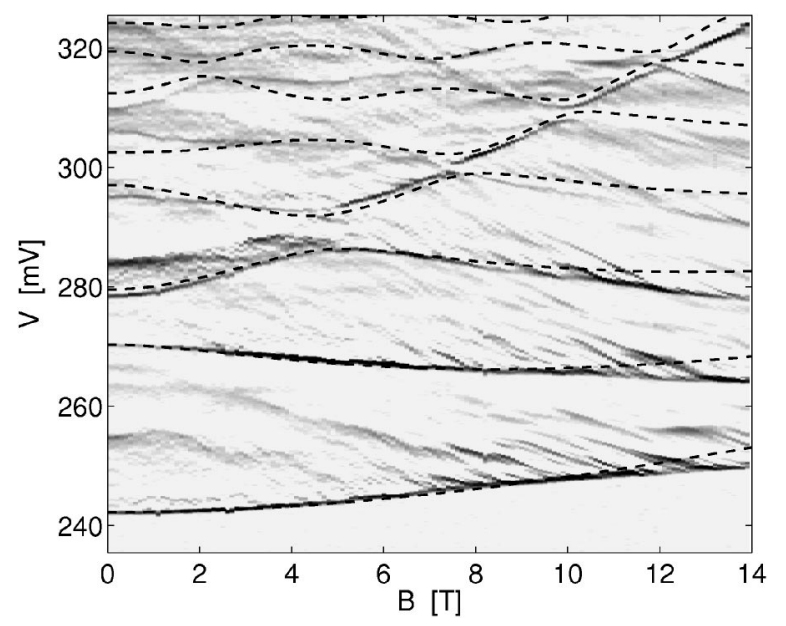

FIG. 4. Measured transport spectrum (repeated from Fig. 2) of a GaAs/AlGaAs QD and the calculated single-electron energies (dashed lines) corresponding to the model potential shown in Fig. 3(a).

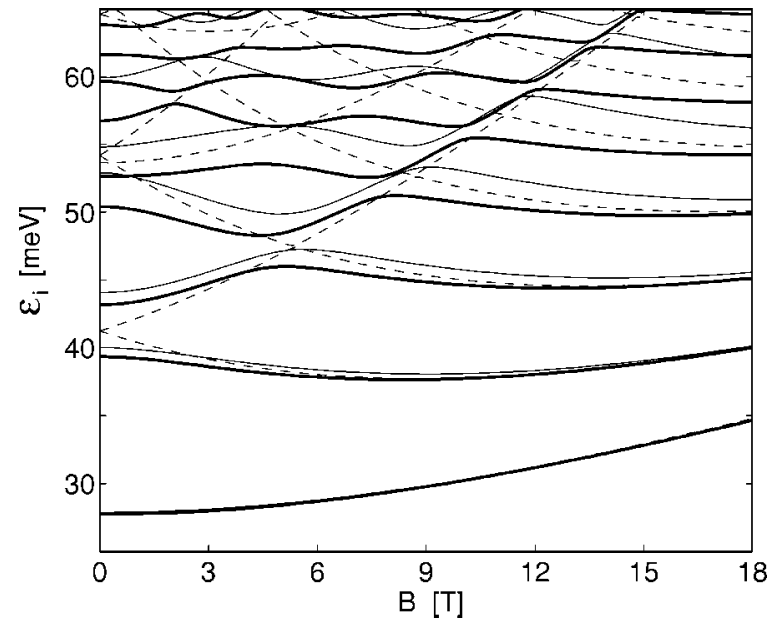

FIG. 5. Lowest noninteracting single-electron eigenenergies calculated for an impurity-containing QD with (thick lines) and without (thin lines) the rounding of the edges. The dashed lines show the corresponding eigenenergies (lifted by $+14 \mathrm{meV}$ for clarity) for a clean dot.

or double-acceptor distorting the QD, possibly a substitutional or interstitial $\mathrm{Si}$ atom migrated through the relatively thin spacer layer; (ii) the impurity is located very close to the QD plane, probably lying in the $10 \mathrm{~nm}$ thick GaAs layer (see Fig. 1). This could lead to the above mentioned reduced value for the real $\epsilon$; (iii) the confinement strength $\hbar \omega_{0}$ is approximately three times larger than the values typically used (3-5 meV) for modeling parabolic QD's. This is due to the growth-induced formation of the QD in the absence of gates around the sample; and (iv) for the same reason, the confinement becomes softer toward the edges of the dot. Hence, the rounding at $r \geqslant r_{c}$ in $V_{\text {conf }}$ is required to compress the highest states in agreement with the experimental spectrum.

We remark that until now we have analyzed several samples showing a, Fock-Darwin-like spectrum ${ }^{6}$ and found only two spectra with clear level repulsion. In this paper, we focused on this particular sample due to the high quality of the transport spectrum.

\section{SHAPE DEPENDENCE}

To clarify the sensitivity of the single-electron spectrum on the shape of the model potential, we compare in Fig. 5 the eigenenergies given by the chosen model (thick lines) to those of a model QD without the rounded edges (thin lines) and to those of a corresponding clean model dot without $V_{\text {imp }}$ but with the rounding term (dashed lines). The level repulsion is clearly induced by the Coulombic impurity that breaks the circular symmetry of the QD and couples the configurations corresponding to different levels. A statistical analysis of the energy-level spacings would enlighten the quantum chaotic properties ${ }^{17}$ of the system but it is not included in this study. It should be noticed that the sequence of the avoided crossings seen in the spectrum (Figs. 4 and 5) is determined by the distance of the distortion from the QD center. In the high-field limit, however, the system becomes 
integrable and the eigenstates condense into Landau levels.

The rounding term in $V_{\text {conf }}$ has the strongest influence on the levels with the highest angular momenta, and the cusp at $r_{c}$ induces also a weak decoupling of the degeneracies at $B$ $=0 \mathrm{~T}$. We remark that the eigenenergies for the clean case are lifted in Fig. 5 by $14 \mathrm{meV}$ for clarity. The Coulombic impurity in the vicinity of the QD thus has a strong effect on the eigenenergies. This tendency is also apparent in the many-electron properties studied below.

\section{MANY-ELECTRON PROPERTIES}

Next we study situations that the quantum dot described by the best fitting parameters above contains up to six interacting electrons. Even if the many-electron case has not yet been experimentally realized for this particular QD, we find it important to predict how the increasing electron number changes the effects of the impurity on the ground-state properties.

The problem is now described by the $N$-electron Hamiltonian

$$
H=\sum_{i=1}^{N}\left[h_{i}+g^{*} \mu_{B} B s_{z, i}\right]+\sum_{i<j}^{N} \frac{e^{2}}{4 \pi \epsilon_{0} \epsilon\left|\mathbf{r}_{i}-\mathbf{r}_{j}\right|},
$$

where the single-electron part $h_{i}$ is given by Eq. (2) and the Zeeman energy is taken into account with $g^{*}=-0.22$ for the effective gyromagnetic ratio in GaAs. This has been measured to be a realistic value for a similar system. ${ }^{18}$

We apply the SDFT according to the self-consistent Kohn-Sham (KS) scheme to obtain the total energies and spin densities of the system. The local spin-density approximation used for the exchange-correlation energy is based on the magnetic-field-independent formulation by Attaccalite $e t$ al. ${ }^{19}$ According to our experience, it is the most accurate parametrization for finite 2D electron systems in the zerofield limit. ${ }^{20}$ We solve the discretized KS equations in real space without implicit symmetry restrictions, which allows us a total freedom in shaping the external potential. The numerical process of solving the effective single-electron Schrödinger equation is accelerated with the Rayleigh quotient multigrid method. ${ }^{16}$

Our earlier calculations for rectangular ${ }^{21}$ and parabolic ${ }^{20}$ QD's show that our SDFT scheme produces energies in a good accordance with the quantum Monte Carlo results. We have also noticed that the current-spin-density-functional theory (CSDFT) does not represent a qualitative improvement over the SDFT in small quantum-dot systems. A detailed comparison between the SDFT and CSDFT for a sixelectron QD can be found in Ref. 20.

\section{A. Energies}

Figure 6 shows the chemical potentials $\mu(N)=E(N)$ $-E(N-1)$, calculated in clean (dashed lines) and impuritycontaining (solid lines) QD's up to six electrons. Due to the spin-degeneracy, the chemical potentials shift generally in adjacent pairs with increasing $B$ corresponding to the wellknown even-odd effect of experimental current peaks. ${ }^{7}$ In the

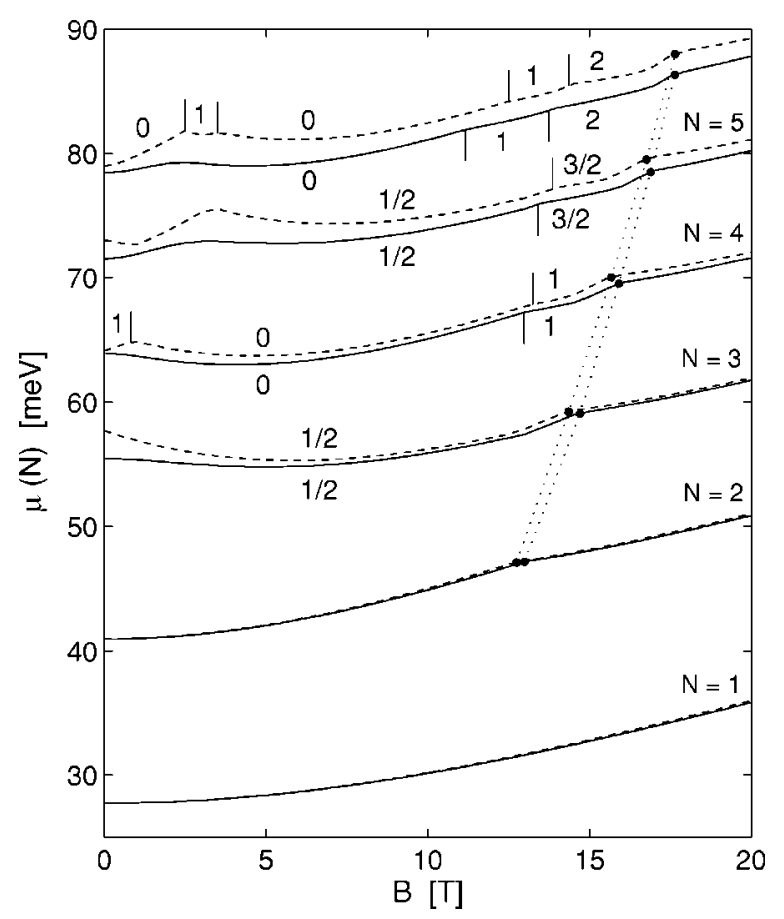

FIG. 6. Calculated chemical potentials for a clean (dashed lines, lifted by $+14 \mathrm{meV}$ for clarity) and impurity-containing (solid lines) quantum dot up to six electrons. The dotted lines denote the borders for the full spin polarization. The other spin-state domains are also marked above and below the curves corresponding to the clean and impurity-containing cases, respectively.

clean case, however, occasional pairing of $\mu(N+1)$ and $\mu(N-1)$ is clearly observable, e.g., with $N=3$ and 5 , and with $N=4$ and 6 at $B \leqq 1 \mathrm{~T}$. This is caused by Hund's rule, i.e., near a degenerate point it is energetically favorable to have parallel spins between the electrons due to the exchange interaction. This leads to partial spin polarization $(S=0$ $\rightarrow 1$ ) in the clean dot with $N=4$ and $N=6$ at $B \leqslant 0.84 \mathrm{~T}$ and $2.5 \mathrm{~T} \leqslant B \leqslant 3.5 \mathrm{~T}$, respectively. In the impurity-containing dot those states are missing due to the avoided level crossings in the single-electron spectrum (see Fig. 5). Generally, the impurity smoothes the behavior of the chemical potential, reflecting the flattening of the single-electron energies studied above. This is in agreement with the results by Güçlü $e t$ $a l .{ }^{11}$ for randomly distributed impurities.

As seen in Fig. 6 the impurity does not affect considerably the onset for the full spin polarization (dotted lines). As $N$ increases, however, this point in the clean QD shifts to higher $B$ than in the impurity-containing dot. Simultaneously, the $\mu$ spacings decrease relatively more rapidly in the latter case than in the clean dot. The reason is the fact that the impurity pushes the electrons to the soft-confinement region near the edges, whereas the clean dot represents a more compact state with higher addition energies.

\section{B. Angular momentum}

In the description above, the impurity is kept in a fixed position at $R=14.5 \mathrm{~nm}$ laterally from the dot center. Next, we consider changes in the total angular momentum as $R$ is 


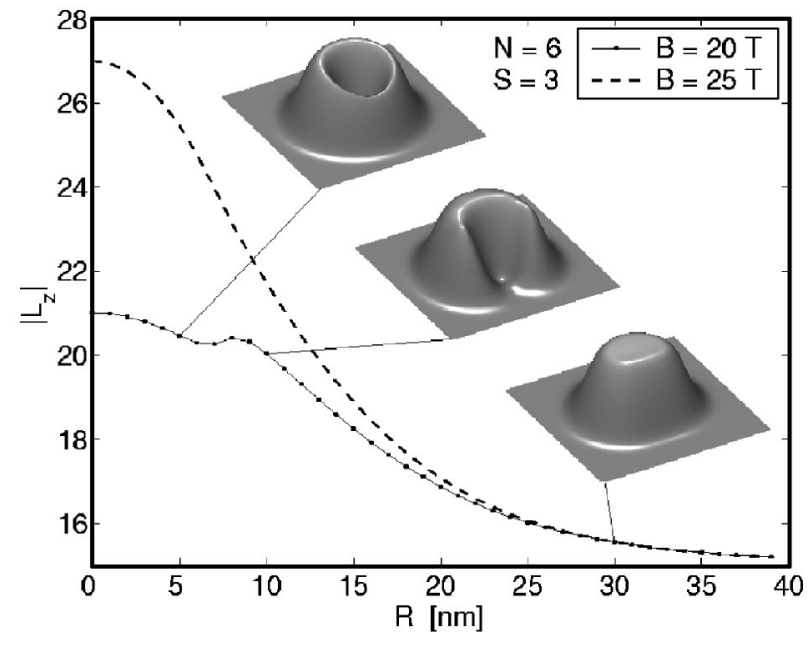

FIG. 7. Total angular momentum and electron density distributions for a six-electron QD as a function of the impurity distance from the dot center. The solid and dashed curves correspond to the magnetic fields of 20 and $25 \mathrm{~T}$, respectively.

varied (while $d$ is kept constant) in magnetic fields corresponding to the MDD state. In a circularly symmetric QD, the MDD has the occupation on the single-particle states with angular momentum eigenvalues $l=0,-1, \ldots,-N+1$, giving $L_{z}=-\frac{1}{2} N(N-1)$ for the total angular momentum. ${ }^{22}$ In the case of a broken circular symmetry, the onset for the MDD can be defined from the last cusp in the chemical potential indicating the full spin polarization (see Fig. 6). This is consistent with the experimental identification of the MDD phase by measuring the magnetic field evolution of the Coulomb blockade peaks. ${ }^{23}$ Since the angular momentum $l$ is not a good quantum number for a noncircular confinement, we compute $L_{z}$ in the following as a sum of the expectation values for the single-electron angular momentum operator, $\hat{l}_{z}=-i \hbar[x(\partial / \partial y)-y(\partial / \partial x)]$. The summation is over all the occupied KS states.

In Fig. 7 we present how the six-electron MDD at $B$ $=20 \mathrm{~T}$ is affected by the impurity shifted through the whole QD region. Except for the kink at $R \sim 8 \mathrm{~nm}$, corresponding to the radius at which the impurity penetrates the edge of the QD, $\left|L_{z}\right|$ increases smoothly from 15 to 21 , i.e., from the MDD to a quantum ring with $l=-1, \ldots,-6$ states occupied. The stability is also visualized in the density insets of Fig. 7 corresponding to different impurity locations. The dashed line shows the equivalent evolution at $B=25 \mathrm{~T}$, still corresponding to the MDD region in the clean dot. As $R \rightarrow 0$, however, we find a transition to the next quantum-ring state with $l=-2, \ldots,-7$ states occupied. The rounded edges of the QD clearly make the ring-like states sensitive to the magnetic field. In contrast, the MDD remains as a rather compact state spread across the highly confined central region as the magnetic field is varied.

\section{Magnetization}

Finally, we investigate the tendency of the many-electron structure to screen the external impurity. For that purpose,

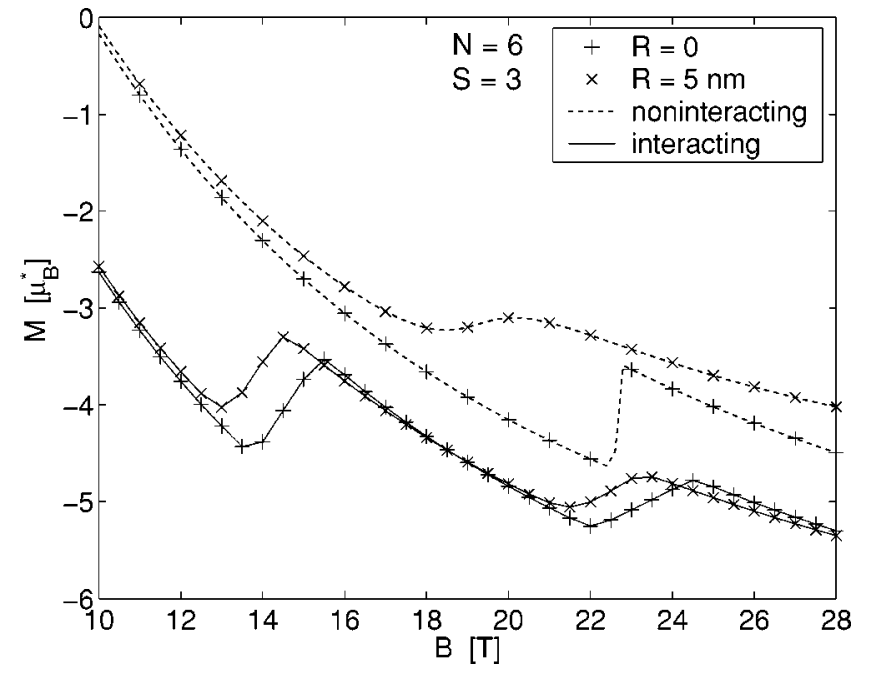

FIG. 8. Magnetization of the (fully polarized) state of a sixelectron quantum dot at the impurity distances $R=0$ (plus markers) and $5 \mathrm{~nm}$ (cross markers) with (solid curves) and without (dashed curves) the electron-electron interactions. The effective Bohr magneton $\mu_{B}^{*}=e \hbar / 2 m^{*}$.

we consider the total magnetization, defined at zero temperature as $M=-\partial E_{\mathrm{tot}} / \partial B$. In Fig. 8 we plot $M$ as a function of $B$ for six-electron, fully polarized QD's in both noninteracting and interacting cases and with the impurity locations $R=0$ and $5 \mathrm{~nm}$ laterally from the QD center. The noninteracting energy is computed directly as a sum of the six lowest single-electron energies. As the impurity is shifted off the dot center, the lowest single-electron states decouple (see Fig. 1 in Ref. 3), leading to a considerably smaller $|M|$ and disappearance of the transition at $B \sim 23 \mathrm{~T}$ in the noninteracting systems. On the other hand, the interacting systems show frequent oscillations due to the entanglement of the energy levels, i.e., periodic changes in the fully-polarized ground state. ${ }^{14}$ The QD's with different impurity locations behave similarly, indicating that the circular properties are preserved also when the impurity is off-center. This verifies the screening of the impurity by the electrons. The importance of screening is evident also from the work by Halonen et al. ${ }^{3}$ who considered impurity-containing QD's with two and three electrons. The same tendency was also found in the magnetization of the two-electron square QD's studied by Sheng and $\mathrm{Xu}^{24} \mathrm{In}$ our forthcoming studies, we will examine the high magnetic field regime as a function of the impurity parameters in more detail.

\section{SUMMARY}

We have reported a transport measurement of a vertical quantum dot, giving single-electron energies strongly deviating from the Fock-Darwin spectrum. We have found a realistic model for the sample dot by including a Coulombic impurity in the external parabolic potential that has soft edges. The parameters obtained for the model potential indicate a strong confinement resulting from the growth-induced formation of the QD, as well as an additional impurity particle, presumably a single or doubly charged $\mathrm{Si}$ atom, shifted 
both laterally and vertically from the center of the quantum dot. Using the model and the spin-density-functional theory, we have studied the energetics and structural properties of the corresponding many-electron problem. The impurity evens out the state alternation as a function of the magnetic field by lifting the degeneracies, but it does not affect remarkably the onset of the full spin polarization. The stability of both the maximum-density droplet and the magnetization verify that the many-electron structure reduces the distortion apparent in the single-electron properties. The future experiments may illustrate the damping of the irregular behavior in actual quantum-dot devices as the number of electrons increases.

\section{ACKNOWLEDGMENTS}

This research was supported by the Academy of Finland through its Centers of Excellence program (2000-2005). E.R. is also grateful for the Magnus Ehrnrooth Foundation for financial support and A. Harju and A. D. Güçlü for useful discussions. The group in Hannover acknowledges sample growth by V. Avrutin and A. Waag and financial support by BMBF.
*Electronic address: ehr@fyslab.hut.fi

${ }^{1}$ For a review, see, e.g., L. P. Kouwenhoven, D. G. Austing, and S. Tarucha, Rep. Prog. Phys. 64, 701 (2001); S. M. Reimann and M. Manninen, Rev. Mod. Phys. 74, 1283 (2002).

${ }^{2}$ V. Fock, Z. Phys. 47, 466 (1928); C. G. Darwin, Proc. Cambridge Philos. Soc. 27, 86 (1930).

${ }^{3}$ V. Halonen, P. Hyvönen, P. Pietiläinen, and T. Chakraborty, Phys. Rev. B 53, 6971 (1996).

${ }^{4}$ P. L. McEuen, E. B. Foxman, U. Meirav, M. A. Kastner, Y. Meir, N. S. Wingreen, and S. J. Wind, Phys. Rev. Lett. 66, 1926 (1991); J. Weis, R. J. Haug, K. v. Klitzing, and K. Ploog, Phys. Rev. B 46, 12837 (1992).

${ }^{5}$ T. Schmidt, M. Tewordt, R. H. Blick, R. J. Haug, D. Pfannkuche, K. v. Klitzing, A. Förster, and H. Lüth, Phys. Rev. B 51, 5570 (1995).

${ }^{6}$ J. Könemann, D. K. Maude, V. Avrutin, A. Waag, and R. J. Haug, Physica E (Amsterdam) 22, 434 (2004).

${ }^{7}$ S. Tarucha, D. G. Austing, T. Honda, R. J. van der Hage, and L. P. Kouwenhoven, Phys. Rev. Lett. 77, 3613 (1996).

${ }^{8}$ R. C. Ashoori, H. L. Stormer, J. S. Weiner, L. N. Pfeiffer, S. J. Pearton, K. W. Baldwin, and K. W. West, Phys. Rev. Lett. 68, 3088 (1992).

${ }^{9}$ Y. Wan, G. Ortiz, and P. Phillips, Phys. Rev. B 55, 5313 (1997).

${ }^{10}$ E. Lee, A. Puzder, M. Y. Chou, T. Uzer, and D. Farrelly, Phys. Rev. B 57, 12281 (1998).

${ }^{11}$ A. D. Güçlü, J. S. Wang, and H. Guo, Phys. Rev. B 68, 035304
(2003).

${ }^{12}$ K. Hirose and N. S. Wingreen, Phys. Rev. B 65, 193305 (2002).

${ }^{13}$ K.-H. Ahn, K. Richter, and In-Ho Lee, Phys. Rev. Lett. 83, 4144 (1999).

${ }^{14}$ E. Räsänen, A. Harju, M. J. Puska, and R. M. Nieminen, Phys. Rev. B 69, 165309 (2004).

${ }^{15}$ J. Könemann, P. König, and R. J. Haug, Physica E (Amsterdam) 13, 675 (2002).

${ }^{16}$ M. Heiskanen, T. Torsti, M. J. Puska, and R. M. Nieminen, Phys. Rev. B 63, 245106 (2001).

${ }^{17}$ H.-J. Stockmann, Quantum Chaos: An Introduction (Cambridge University Press, Cambridge, 2000).

${ }^{18}$ J. Könemann, R. J. Huag, V. I. Falko, D. K. Maude, and B. L. Altschuler (unpublished).

${ }^{19}$ C. Attaccalite, S. Moroni, P. Gori-Giorgi, and G. B. Bachelet, Phys. Rev. Lett. 88, 256601 (2002); 91, 109902(E) (2003).

${ }^{20}$ H. Saarikoski, E. Räsänen, S. Siljamäki, A. Harju, M. J. Puska, and R. M. Nieminen, Phys. Rev. B 67, 205327 (2003).

${ }^{21}$ E. Räsänen, H. Saarikoski, V. N. Stavrou, A. Harju, M. J. Puska, and R. M. Nieminen, Phys. Rev. B 67, 235307 (2003).

${ }^{22}$ S.-R. Eric Yang and A. H. MacDonald, Phys. Rev. B 66, 041304(R) (2002), and references therein.

${ }^{23}$ T. H. Oosterkamp, J. W. Janssen, L. P. Kouwenhoven, D. G. Austing, T. Honda, and S. Tarucha, Phys. Rev. Lett. 82, 2931 (1999).

${ }^{24}$ W. Sheng and H. Xu, Physica B 256-258, 152 (1998). 